\title{
Identification of Mind-Set of Students through Web based Basic Psychological Text and Graphical Analysis System
}

\author{
Tabassum Rizvi
}

\begin{abstract}
In this era of 21st century the most talked subject regarding children is mind-set. According to Carol Dweck, mind-set can predict success in life. It's an internal voice that keeps you down or lifts you up. It is very important to understand student's mind-set and mental health, so proper care, inputs, facility and necessary arrangement can be taken at very early stage for uplifting career growth. Mind-set of a person may be guessed from the outlook of the person and from the mental status of the person i.e mentality. Mind-set of a person reflects in psyche, attitude, and ethos. Now, the parameters that influenced mind-set of a person are family members with whom they are born and brought up, behaviour of family members, surroundings environment, situation under which they are born and brought up, facilities provided and friends circle etc. There are two types of mind-set basically resides in students one is growth Mind-set and the other is Fixed Mind-set. It has been observed that for last one decade the number of suicide and depression in students at the age group of 15 to 20 years has increased a lot. State of mind-set is not permanent. The parameters affects positive growth of mind-set can be nullified with some practice and change of habits. Right mind-set can be nourished choosing growth over negativity. Though this research paper, attempt was made to identify the different stages of mind-set of students and problems faced by them which hinders students' progress and makes them depressed through web based graphical interactive system by using Gradient and Pearson Correlation Co-efficient method.
\end{abstract}

Keywords: Attitude, ethos, graphical, gradient, Mind-set, psyche, web.

\section{INTRODUCTION}

Web based graphical system is being used to record user inputs based on some attributes selected by the users on the system. The attributes may be the general question, image, psychological theme and voice detection. This system is widely used in computer science data analysis system. The traditional system of consultation is very time consuming and tedious job. It is also depends on the availability of the user as well. In this scenario the same process is repeated for each user. To make the system time saving, cumber some free, remove the complexity a web based system is introduced in this paper. This system can give comparative study and grouping of similar nature and similar mind-set person for further analysis.

Revised Manuscript Received on March 13, 2020.

* Correspondence Author

Dr. Tabassum Rizvi*, Assistant Professor, Department of Political Science, The Assam Royal Global University, (Guwahati), Assam, India.

(c) The Authors. Published by Blue Eyes Intelligence Engineering and Sciences Publication (BEIESP). This is an open access article under the CC BY-NC-ND license (http://creativecommons.org/licenses/by-nc-nd/4.0/)
This system is user-friendly and graphical. The combination of text and graphical based UI provide a sophisticated highly secure system. This technology is efficient way of analysing student's mind-set by considering different angles and perspective of the users considering all condition and status of mind. The system user need not required to remember the series of past transactions and history. Normal system after certain interval of time we need to redo the process and physical file search and record find out is very difficult and hence keeping tract is also difficult. Schools, colleges, research institutes, any organization where this analysis is required to assess the student's mind-set and improve positive growth this system can help extensively to extract sensitive data for analysis. These applications can be accessed at any level and in general can be utilized for all level of users. As a result for ease of use with high level user friendly security system can only be the solution in such case.

The system is developed using correlation technique is very latest and effective technique to provide data output for further analysis based assigned criteria. The correlation technique is convenient to use in this system of data analysis and is very unique. To identify common nature and behaviour clustering and correlation algorithm are used here. The inputs submitted by the user shall be converted to some unique pattern, sampling point and threshold value for every individual. This pattern and values can be classified by analysing and comparing with pattern matching algorithm. In case of image and pattern input this system will extract the image and pattern convert in digital format assign threshold value as per psychological parameter evaluation. And these can easily be matched and compared with the help of pattern matching and clustering technique.

The web based user analysis system is very convenient to use in psychological parameter evaluation system for easy use. In the paper, it is considered to have an image and psychological parameter warehouse for huge collection of images, text, graphical designs based on the analysis required. Once the user select the graphical mode the image warehouse will be shown and the user can select the images and answer the psychological parameter question with suitable work from the select option as desired.

The alphanumeric word and graphical pattern in this case the user can select as per different condition and arrangement made by the psychological evaluation system.This paper illustrates the multilevel highly secure mind set detection, behavior analysis and identification of psychological state analysis system.

\section{Published By:}

Blue Eyes Intelligence Engineering

\& Sciences Publication

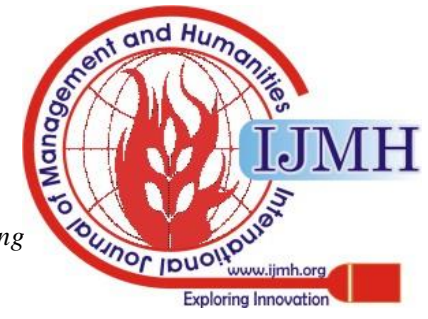




\section{CLUSTERING AND SAMPLE MATCHING TECHNIQUE USING GRADIENT \& PEARSON CO-EFFICIENT METHOD:}

Clustering and sample matching is very efficient way of finding correlation. The input provided by the user analysed and groped using this algorithm.

The steps involved in clustering grouping of correlated data are as followed:

Step1: Web based interface to collect user inputs.

Step2: Assigning values to the corresponding inputs and per psychological parameter.

Step 3: Pre-processing of the values.

Step 4: Formation of sample point.

Step 5: Comparison \& matching.

Step 6: Grouping \& clustering.

Step 7: Final Analysis Result as per the values.

Physical environment: User shall submit their inputs through web based computer interface where graphical, text based answering and writing options available in the system. These data are stored in digital format by assigning some values.

Raw data collection: The raw data collected from the application are arranged in sequential manner in binary format. The binary data is converted to decimal format and with these decimal format sequential data a sample list is formulated for sample based analysis.

Pre-processing of raw data: The collected raw data consists of noise data. The noise data is removed before from the sequential samples. The nosy data can be identified by the comparing nearest sequential data difference. The difference of sequential data is categorized as $+1,0$ and -1 . The data difference beyond this range are identified as noisy data and are removed and rearranged the sample list.

Formation of pattern: The pattern formation is done by finding the slope or gradient of the consecutive points and formed one two dimensional matrix. For example,

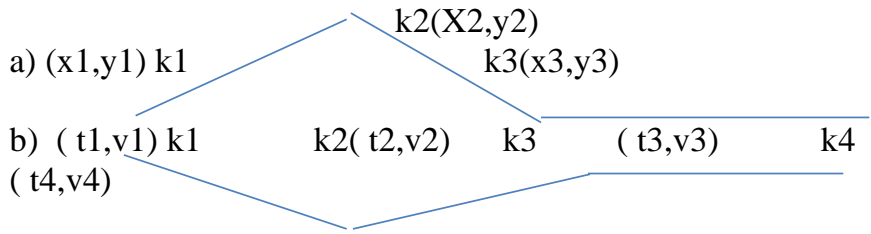

Fig: 1, Two Dimensional Graph

The slope between two coordinates of pattern (a) \& (b) can be calculated as, Gm(slope) $=(\mathrm{Y} 2-\mathrm{Y} 1) /(\mathrm{X} 2-\mathrm{X} 1)$.

The slopes of consecutive coordinates of the patterns is stored in the two dimensional matrix as below

\begin{tabular}{c|ccc} 
& $\mathrm{k} 1$ & $\mathrm{k} 2 \mathrm{k} 3$ & \\
\hline $\mathrm{a}$ & +0.60 & -0.58 & 0 \\
$\mathrm{~b}$ & -0.44 & +0.30 & -0.18
\end{tabular}

Fig: 2, Two Dimensional MATRIX

Comparison, matching and Grouping: The comparison matching and grouping of the patterns can be done with Pearson's Correlation Coefficient Algorithm. Pearson's correlation coefficient measures the linear relations or association between two variables $\mathrm{X}$ and $\mathrm{Y}$, giving a value between +1 and -1 inclusive, where 1 is total positive correlation, 0 is no correlation, and -1 is total negative correlation. This algorithm identifies how much similar are the two variables $\mathrm{X}$ and $\mathrm{Y}$.

The formula for calculating Pearson's correlation coefficient [ $\rho$ (rho) ] is mentioned below:

$\rho_{X, Y}=\frac{\operatorname{cov}(X, Y)}{\sigma_{X} \sigma_{Y}}$

Where:

- cov is the covariance

- $\sigma_{X}$ is the standard deviation of $X$

The formula for $\rho$ can be expressed in terms of mean and expectation.

The covariance can be calculated as below:

$$
\operatorname{cov}(X, Y)=\mathrm{E}\left[\left(X-\mu_{X}\right)\left(Y-\mu_{Y}\right)\right]
$$

The standard deviation of $X$ can be calculated as below: In the case where $X$ takes random values from a finite data set $x_{1}, x_{2}, \ldots, x_{N}$, with each value having the same probability, the standard deviation is

$\sigma=\sqrt{\frac{1}{N}\left[\left(x_{1}-\mu\right)^{2}+\left(x_{2}-\mu\right)^{2}+\cdots+\left(x_{N}-\mu\right)^{2}\right]}$, where $\mu=\frac{1}{N}\left(x_{1}+\cdots+x_{N}\right)$,

Then the formula for $\rho$ can also be written as

$$
\rho_{X, Y}=\frac{\mathrm{E}\left[\left(X-\mu_{X}\right)\left(Y-\mu_{Y}\right)\right]}{\sigma_{X} \sigma_{Y}}
$$

where:

- $\operatorname{cov}$ and $\sigma_{X}$ are defined as above

- $\mu_{X}$ is the mean of $X$

- $\mathrm{E}$ is the expectation.

For the pattern (a) and (b) the Pearson's Correlation k\$pefficient $(\rho)$ value can be calculated as below:

$\mu$ (mean) value for slope samples[Pattern a]: [+0.60 $+(-0.58)+0] / 3=-0.006$

$\mu($ mean) value for slope samples [pattern b]: $[-0.44+0.30+$ $(-0.18] / 3=-0.106$

Standard Deviation for pattern a: $\sigma(\mathrm{a})=\sqrt{1 / 3\left([0.60-(-0.006)]^{2}+[-0.58-(-0.006)]^{2}+[0-(-0.006)]^{2}\right)=0.48}$

Standard Deviation for pattern b: $\sigma(b)=\sqrt{\left.1 / 3[-0.44-(-0.106)]^{2}+[0.30-(-0.106)]^{2}+[-0.18-(-0.106)]^{2}\right)}=0.31$

Hence Pearson's Correlation Coefficient $(\rho)$ for point p1 of pattern $\mathrm{a}$ and $\mathrm{b}$.

$\rho(\mathrm{a}, \mathrm{b})$ for $\mathrm{k} 1=\{0.60-(-0.006)\}\{-0.44-(-0.106)\} /(0.48 \mathrm{x}$ $0.31)=-1.83$

$\rho$ (a,b) for $\mathrm{k} 1<0$, hence part $\mathrm{k} 1$ for $\mathrm{a}$ and $\mathrm{b}$ pattern are negatively co-related.

Published By: 
The calculated Pearson's Correlation Coefficient $(\rho)$ are categorized in the following ranges:

$$
\text { If } \begin{array}{rlr}
\rho<0 \quad & -1 \\
& =0 & 0
\end{array}
$$

$>0 \quad+1$

Considering these ranges the two dimensional matrix can be formulated as mentioned below which called Regulation Matrix is:

\begin{tabular}{cccc} 
& $\mathrm{k} 1$ & $\mathrm{k} 2$ & $\mathrm{k} 3$ \\
\hline $\mathrm{a}$ & -1 & -1 & 1 \\
$\mathrm{~b}$ & -1 & -1 & 1
\end{tabular}

From the above regulation matrix the two patterns (a) and (b) can easily be compared. The two parts k1 \& k2 of pattern (a) and (b) have negative correlation and part k3 has positive correlation i.e k3 of both patterns is similar.

\section{THE ALGORITHM}

\section{a) User Input Graphical Representation}

1) Psychological parameters, images etc. Storing.

2) All parameters are assigned threshold values.

3) These threshold values are converted to sample points considering suitable units.

4) Drawing two dimensional graph using JAVA based programming with these sample points [Fig.1].

\section{b) Gradient Calculation}

1) From two dimensional graph for each sample points $(\mathrm{x}, \mathrm{y})$ or-ordinated are identified and gradients are calculated.

2) Storing of gradient in two dimensional MATRIX.[Fig.2]

3) This process is repeated for all graphs and gradient values are stored in MATRIX. [Fig.2]

c) Comparison, matching and Grouping

1) Calculating Mean values for all patterns.

2) Calculation of Standard Deviation of all patterns.

3) Calculation of Pearson's Correlation Coefficient for

4) Based on Pearson's Correlation Coefficient value assign ranges and store in two dimensional matrix.

5) Based on the ranges patterns are compared and which are positively co-related and which are negatively co-related are identified and grouped respectively.

6) These results to identify the similar mind set and similar psychological behaviour of students' statistics for further study.

\section{CONCLUSION}

The objective of this paper is to build a comparative study web based system which is secure and user friendly. The system designed here to provide students different aspects like status of mindset, behaviour based on some psychological inputs. It has been observed that in today's world the most difficult thing is to make students adaptive, efficient in all fields, tension and stress free, gaining concentration level, pro-activeness and stable from mind. Considering environment and competitiveness in the world the conventional way of improving child's skill is helping in this era. It is difficult to remember for all things for students and hence based on the capacity, similar nature and strength each sample points.

students should be treated in the similar way and similar procedures can be applied. The web based system is very unique and provide comprehensive data set for study students' nature. This is very secure system and authentication is restricted for information and data safety. The process is very simple using some psychological parameters and images the user inputs are collected. For each inputs threshold values are assigned these values are converted into sample points with suitable units.

These sample point are converted to two dimensional graphs. The algorithm designed here is implemented by using the concept of gradient and Pearson co-relation method. The gradient concept is used here to form patterns and the similarities of these patterns are identified by using the Pearson co-relation method.

Every set of value of graphical pattern is stored in two dimensional matrix. Checking, grouping, matching and identification can easily be done simple comparison algorithm. The system developed give idea of comparative study of common parameters of students and how they can be treated. There is always a scope for improvement of any algorithm designed, how efficient and fruitful the system may be. The most important aspect of the system is that how flexible is the system to adapt the future modifications. This system has factored into different sections and modules to adapt any further modifications. All possible efforts has been put forward to make system efficient and user-friendly.

\section{ACKNOWLEDGMENT}

I would like to thank my colleagues and friends from the Department of Political Science of the Assam Royal Global University who have extended their commendable and unwavering help for the completion of this paper.

\section{REFERENCES}

1. Hoque Soriful : "Multilevel and Biometric-Graphical Secure Authentication System Using Pattern Matching and Gene Based Data"

2. Banfield J. D. and Raftery A. E.: "Model based Gaussian and non-Gaussian clustering", Biometrics 49, September 1993, pp. 803-821.

3. Byers S. and Raftery A. E.: "Nearest Neighbor Clutter Removal for Estimating Features in Spatial Point Processes", Technical Report No. 305, Department of Statistics, University of Washington. [Available at http://www.stat.washington.edu/tech.reports/tr295.ps]

4. Ester M., Kriegel H.-P., Sander J., Xu X.: "A Density- Based Algorithm for Discovering Clusters in Large Spatial Databases with Noise", Proc. 2cnd Int. Conf. on Knowledge Discovery and Data Mining, Portland, Oregon, 1996, AAAI Press, 1996

5. Ester M., Kriegel H.-P., Xu X.: "Knowledge Discovery in Large Spatial Databases: Focusing Techniques for Efficient Class Identification", Proc. 4th Int. Symp. On Large Spatial Databases, Portland, ME, 1995, in: Lecture Notes in Computer Science, Vol.951, Springer, 1995, pp.67-82.

6. Fayyad U. M.,.J., Piatetsky-Shapiro G., Smyth P.: "From Data Mining to Knowledge Discovery: An Overview", in: Advances in Knowledge Discovery and Data Mining, AAAI Press, Menlo Park,1996, pp. 1 - 34

7. Gueting R. H.: "An Introduction to Spatial Database Systems", in:The VLDB Journal, Vol. 3, No. 4, October 1994, pp.357-399.

8. Kaufman L., Rousseeuw P. J.: "Finding Groups in Data: An Introduction to Cluster Analysis", John Wiley \& Sons, 1990.

9. McKenzie M., Miller R., and Uhrhammer R.: "Bulletin of the Seismographic Stations", University of California, Berkeley. Vol.53, No. 1-2.

\section{Published By:}


10. Muise R. and Smith C.: "Nonparametric minefield detection and localization", Technical Report CSS-TM- 591-91, Naval Surface Warfare Center, Coastal Systems Station.

\section{AUTHORS PROFILE}

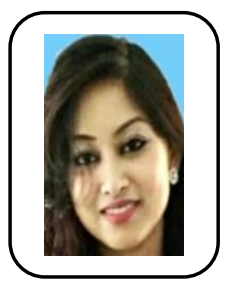

Dr. Tabassum Rizvi, Assistant Professor, Department of Political Science, The Assam Royal Global University Assam, India. Research interest in the areas like Indian Government and Politics, Women Studies, Role of Information and Communication Technology and the Social Science Research, etc. I have presented research papers in national, international seminars and published articles in national and international journals. 